\title{
Triple Noncontiguous Thoracic Spine Fractures Treated with Hybrid 3D Intraoperative CT- Navigated Minimal Invasive Fixation Technique: A Case Report
}

ISSN: 2637-7748

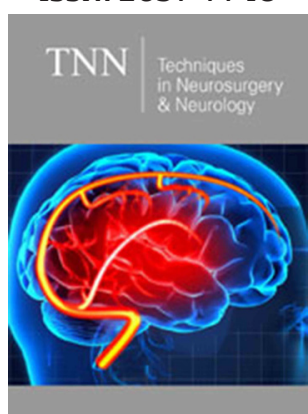

*Corresponding author: Patrick Misson, C.H.U. of Charleroi, University of Liège, Belgium

Submission: 侮June 01, 2020

Published: 㘹June 12, 2020

Volume 3 - Issue 3

How to cite this article: Patrick Misson, Abdelhammid Mendili, Corentin Delhaye, et al. Triple Noncontiguous Thoracic Spine Fractures Treated with Hybrid 3D Intraoperative CT-Navigated Minimal Invasive Fixation Technique: A Case Report. Tech Neurosurg Neurol. 3(3). TNN 000563. 2020.

DOI: $10.31031 /$ TNN.2020.03.000563

Copyright@ Patrick Misson, This article is distributed under the terms of the Creative Commons Attribution 4.0 International License, which permits unrestricted use and redistribution provided that the original author and source are credited.

\section{Patrick Misson ${ }^{1 *}$, Abdelhammid Mendilii ${ }^{1}$, Corentin Delhaye ${ }^{2}$, Jean Pirson ${ }^{3}$,} Didier Martin ${ }^{4}$ and Cristo Chaskis ${ }^{1}$

${ }^{1}$ Department of Neurosurgery, C.H.U. of Charleroi, Belgium

${ }^{2}$ Department of Radiology, C.H.U. of Charleroi, Belgium

${ }^{3}$ Department of Anesthesiology, C.H.U. of Charleroi, Belgium

${ }^{4}$ Department of Neurosurgery, C.H.U. of Liège, University of Liège, Belgium

\section{Abstract}

Introduction: Multilevel noncontiguous thoracic spine fractures are usually due to high-energy trauma and often associated with life threatening organ injuries or neurological deficits. Early posterior percutaneous fixation has shown its efficacy in non-neurologic fractures and for comorbidity management. However, it remains a tricky procedure due to spine instability and anatomic landmark modification. Additional intraoperative CT-assisted navigation might improve precision in such long and complex spine fixation.

Case Presentation: A 26-year old men was admitted to ICU after a very high velocity motorcycle accident at $250 \mathrm{~km} / \mathrm{h}$. Surprisingly, he had no neurological deficits, but the initial traumatic assessment revealed triple unstable noncontiguous thoracic spine fractures of Th4, Th6 and Th8. The patient was treated with a hybrid minimal invasive approach using an intraoperative 3D CT-scan navigation system and percutaneous pedicle-screwing method. The postoperative CT-Scan shown instrumentation from Th2 to Th10 and demonstrated correction of regional kyphosis. The patient regained his mobility and started to walk 3 days following the surgery. He was discharged with temporary additional thoraco-lumbar corset 7 days after surgery without any postoperative complications.

Discussion: Navigated trans-pedicular screw fixation has proven its superiority in precision. The use of live intraoperative CT-scan with spinous process reference marker provides critical information about spine alignment and pedicular trajectories which facilitate screw placement in fractured or misplaced vertebrae. The addition of standard lateral C-arm can be easily implemented.

Conclusion: Noncontiguous triple fractures of the thoracic spine are an unusual presentation of polytrauma injury. Long and complex thoracic posterior spine fixation is feasible in a safe way using an intraoperative CT-navigated minimal invasive technique in support.

Keywords: Noncontiguous spine fractures; Thoracic spine fixation; Minimal invasive technique; Intraoperative CT-scan navigation

Abbreviations: CT: Computed Tomography; ISS: Injury Severity Score; ICU: Intensive Care Unit; ASIA: American Spinal Injury Association; MRI: Magnetic Resonance Imaging; AO-Spine: Arbeit gemeinschaft für Osteo-synthesefragen Spine.

\section{Introduction}

Multilevel noncontiguous thoracic spine burst fractures are rarely reported in the literature [1-5]. They are usually described as floating spine injuries. The reported incidence of noncontiguous spine fractures varies from 1.6 to 19\%, with a usual range of 3 to $8 \%$. However, this incidence has been historically underestimated due to overlooked injuries or delayed X-rays diagnosis. Recent evidences based on routine full-spine CT-scan suggest the actual frequency may be as high as $20 \%$ [6]. These injuries are strongly associated with high-energy trauma, mainly fall from high height or traffic accident [7]. The average age at the time of accident is 30,9 years. They are frequently associated with life threatening organ injuries and neurological deficit, resulting in high Injury Severity Score (ISS) [3,4,6,7]. In such 
clinical situations, early surgical fixation has shown its efficacy compared to conservative treatment. It allows better comorbidity management and leads to better clinical outcomes in terms of mobilization. Recently, percutaneous posterior spinal fixation has become the new gold standard compared to a conventional open approach. It reduces the invasiveness of the procedure and offers better precision [8]. However, percutaneous fixation on multilevel traumatic injuries remains a tricky procedure due to spine instability and anatomic landmark modification. This is particularly the case at thoracic spine level where anatomical variability and the restricted size of the pedicles are limiting the margin of error $[8,9]$. Additional intraoperative CT-assisted navigation might improve precision in such long and complex spine fixation procedure [814]. In the light of the above, we report here the case of a patient with traumatic noncontiguous triple burst fractures of the thoracic spine. To our knowledge, this is the first report of such a long thoracic spine fixation using 3D intraoperative CT-navigated minimal invasive technique for multilevel traumatic fractures. The purposes of this report are twofold. First, it exposes our technical report in this specific case. Secondly, we want to discuss the benefits of intraoperative navigation assistance.

\section{Case Presentation}

A 26-year old man was admitted to ICU after a very high velocity motorcycle accident on a speed track. He has been ejected from his motorbike at a speed of $250 \mathrm{~km} / \mathrm{h}$ and hit the tire wall directly with the back. On the hospital admission, he did not show any major abdominal injury but a respiratory insufficiency due to a bilateral hemopneumothorax requiring bilateral pleural drain. Initial neurological evaluation revealed severe back pain but no neurologic deficit. According to the American Spinal Injury Association (ASIA) impairment scale, his neurological status has been estimated as ASIA E. A crush syndrome was also present at the admission but resolved within 24 hours with optimal hydration and urine alkalization.

\section{Radiologic Findings}

The initial whole-body computed tomography revealed three unstable noncontiguous vertebral body fractures distributed on Th4, Th6 and Th8. These were complicated by the association of multiple fractures of pedicles, spinous and transverse processes at adjacent thoracic spine levels (Figure1). These fractures were graded according to AO Spine classification as it follows: type A4 with $32 \%$ vertebral compression on Th4; type A3 with $18 \%$ vertebral compression on Th6; type B2 with 75\% vertebral compression on Th8 (Table 1). The regional kyphosis between Th2 and Th10 was measured with an angle of $38.5^{\circ}$. The computed tomography also revealed a fracture of the sternal manubrium, multiple bilateral rib fractures from Th5 to Th9 and a left great trochanter fracture. Interestingly the whole spine MRI assessment did not show any spinal cord injury neither epidural lesions. The ISS was measured at 24 .

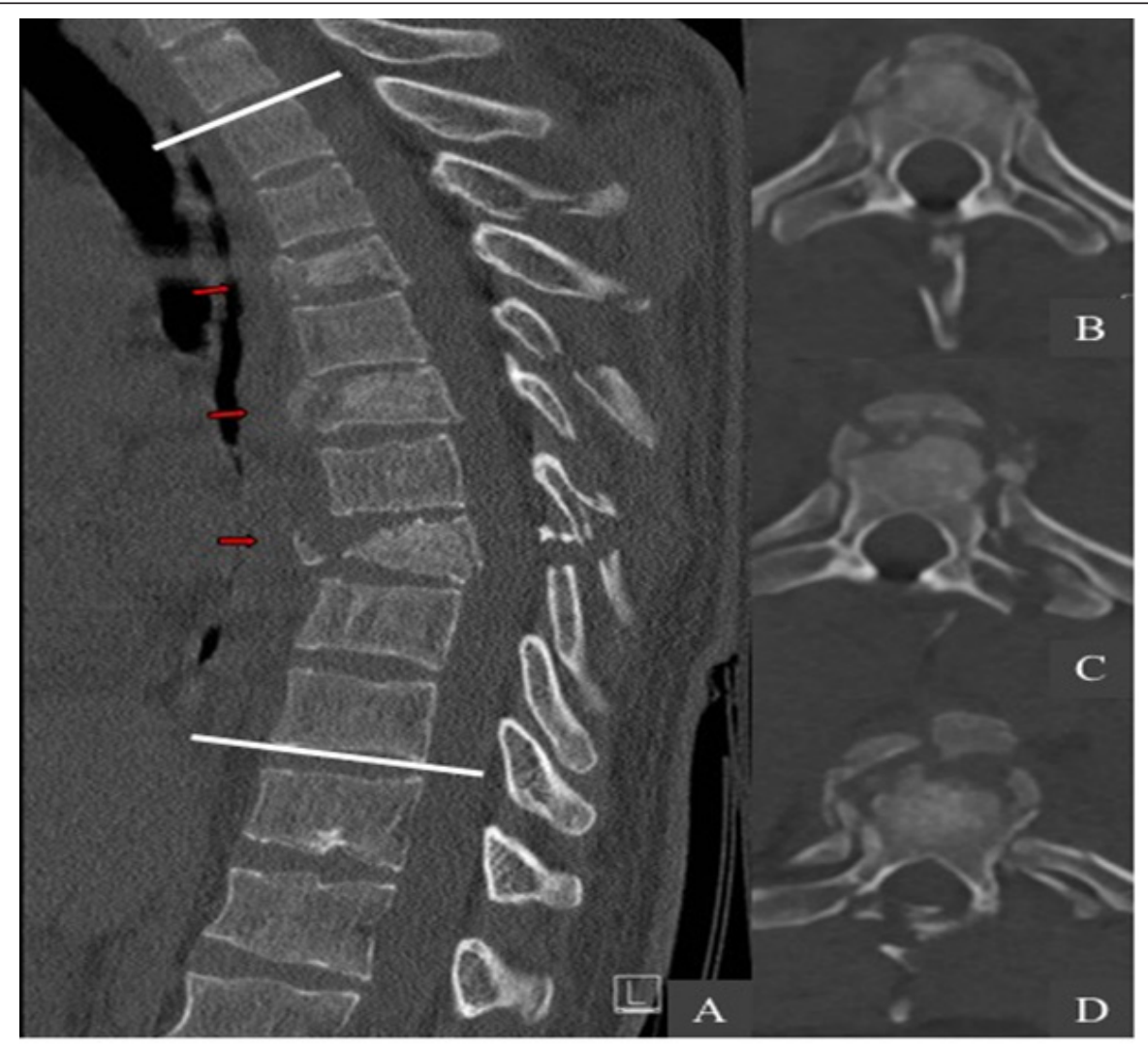

Figure 1: Preoperative CT-scan. (A) The sagittal and axial views show three unstable noncontiguous vertebral body fractures (red arrows) distributed on Th4 (B), Th6 (C) and Th8 (D). The regional kyphosis was measured with an angle of $38.5^{\circ}$ between Th2 and Th10 (white lines). 
Table 1: Fractures summary according to the AO Spine classification.

\begin{tabular}{|c|c|}
\hline Level & Fractures Types \\
\hline Th3 & Spinous process \\
\hline Th4 & Burst fracture type A4, spinous process \\
\hline Th5 & Bi-pedicular, left transverse process, spinous process \\
\hline Th6 & Burst fracture type A3, right transverse process, spinous process \\
\hline Th7 & Left pedicle, left transverse process, spinous process \\
\hline Th8 & Burst fracture type B2, bilateral transverse processes, spinous process \\
\hline Th9 & Left transverse process \\
\hline
\end{tabular}

\section{Surgical Technique}

The patient undergone a surgery based on a hybrid minimal invasive approach using an intraoperative 3D CT-scan navigation system and percutaneous pedicle-screwing method. We performed a posterior thoracic spine fixation from Th2 to Th10. The patient was placed in a prone position on a mobile, radiolucent, carbon fiber operating table connected to the Airo ring (Figure 2A). A fluoroscopy was used to check the spinal alignment and to determine the vertebral levels to be instrumented. A medial posterior skin incision was performed from Th1 to Th11 down to the muscular fascia. At this stage, a navigated reference marker clamp was firmly placed with minimal muscle dissection on the spinous process of Th9, which was the closest intact bony structure adjacent to the fractured levels [10] (Figure 2B). A first intraoperative CT-scan acquisition was performed, assessing a good kyphosis correction (Figure 3). The acquired datas were processed thanks to the intraoperative image-guidance system. A series of trans-pedicular channel was performed from Th10 to Th7, based on the 3D navigated CT-scan with a navigated standard Jamshidi needle (Figure 4) K-wires were inserted into the pedicles before insertion of cannulated screws. Additional lateral fluoroscopy was performed at the end of each screw placement in order to verify the screwing accuracy and the absence of bone displacement [12]. A second series of pedicular screwing was then realized using the same procedure under the control of a second CT-scan with a Th4 reference marker clamp. Two $24 \mathrm{~cm}$ slightly bent titanium rods were placed in a strictly minimal invasive way to connect the screws. A vertebral distraction was applied to the vertebral body of Th8 to restore correct vertebral height and complete the procedure. Further decompressive laminectomy was unnecessary, as the MRI did not highlight any spinal cord compression.

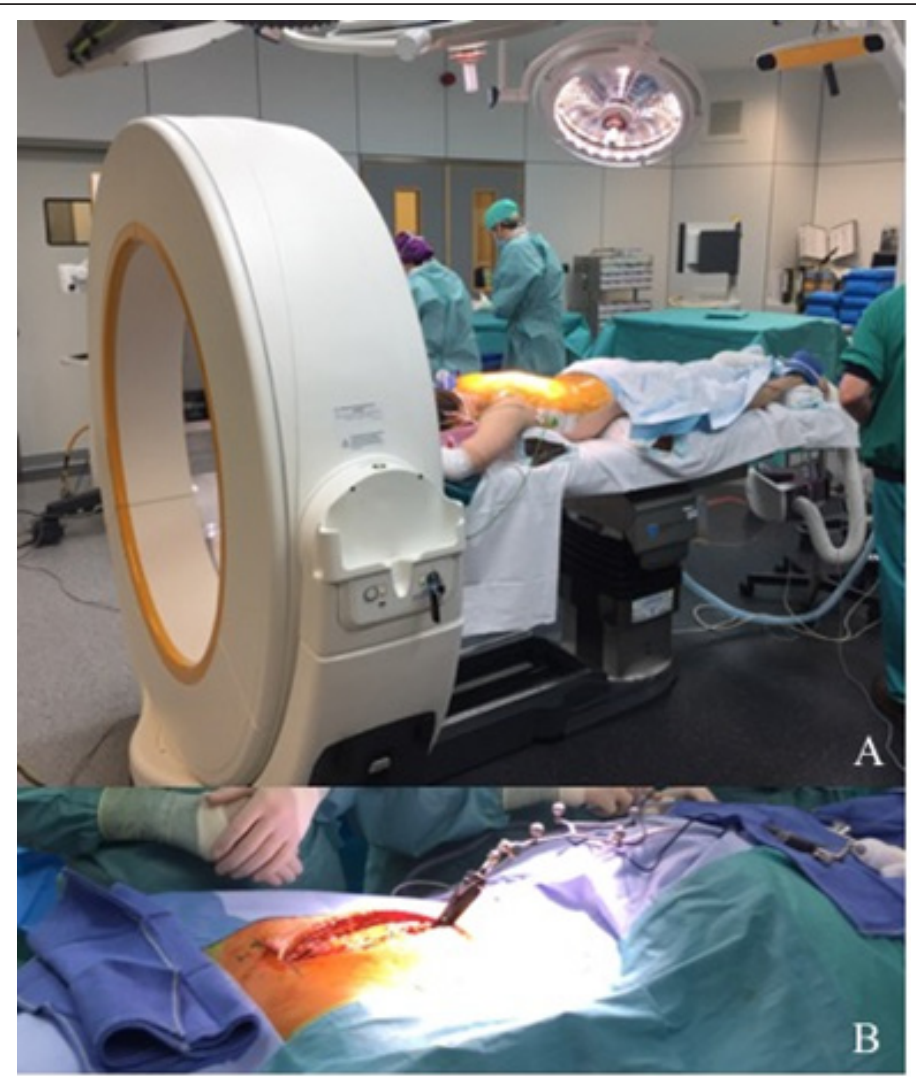

Figure 2: Operating room setting with the Airo ring. (A) Midline incision with navigated reference marker fixed on Th9. 


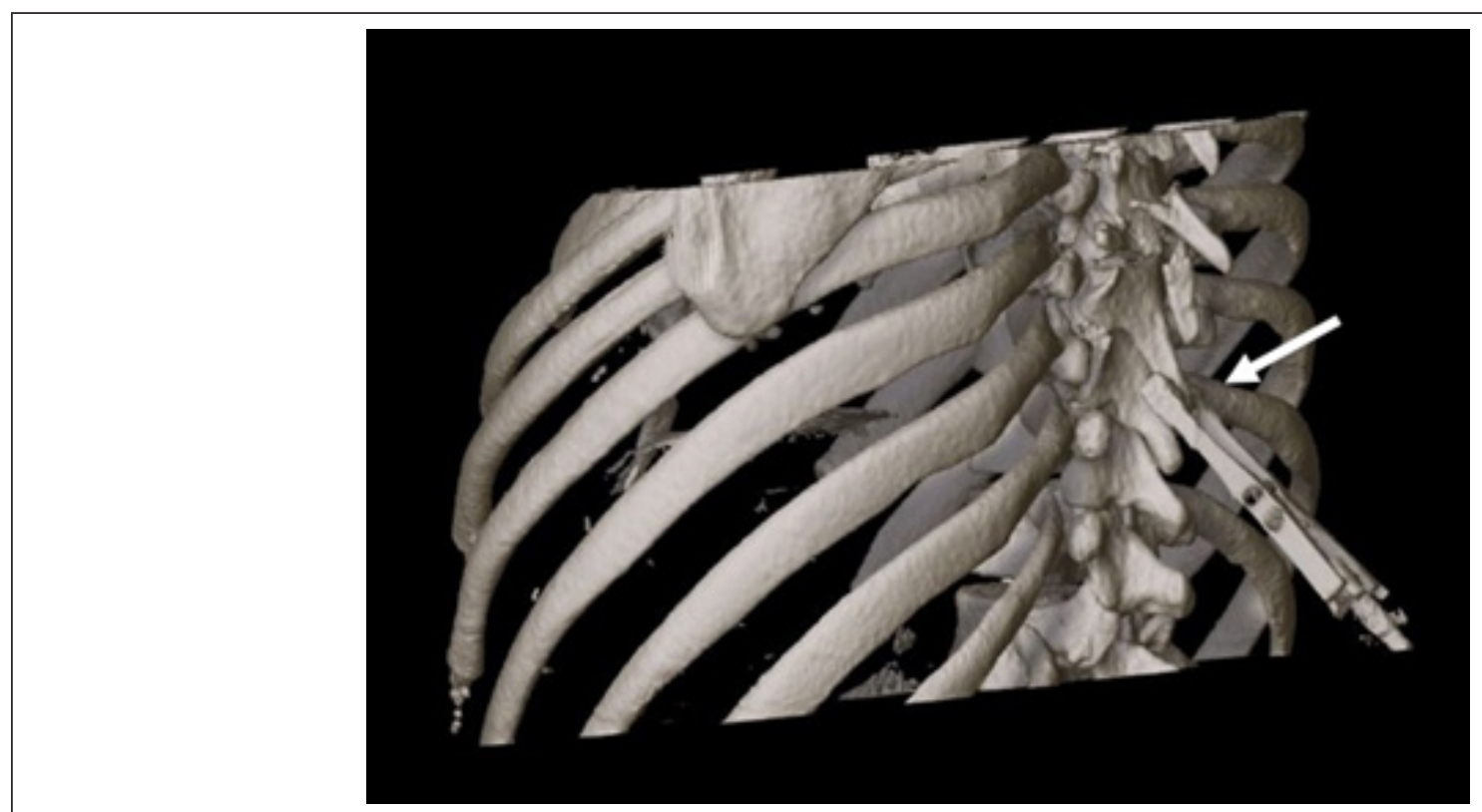

Figure 3: First intraoperative CT-scan acquisition with reference marker fixed on the Th9 spinous process (white arrow).

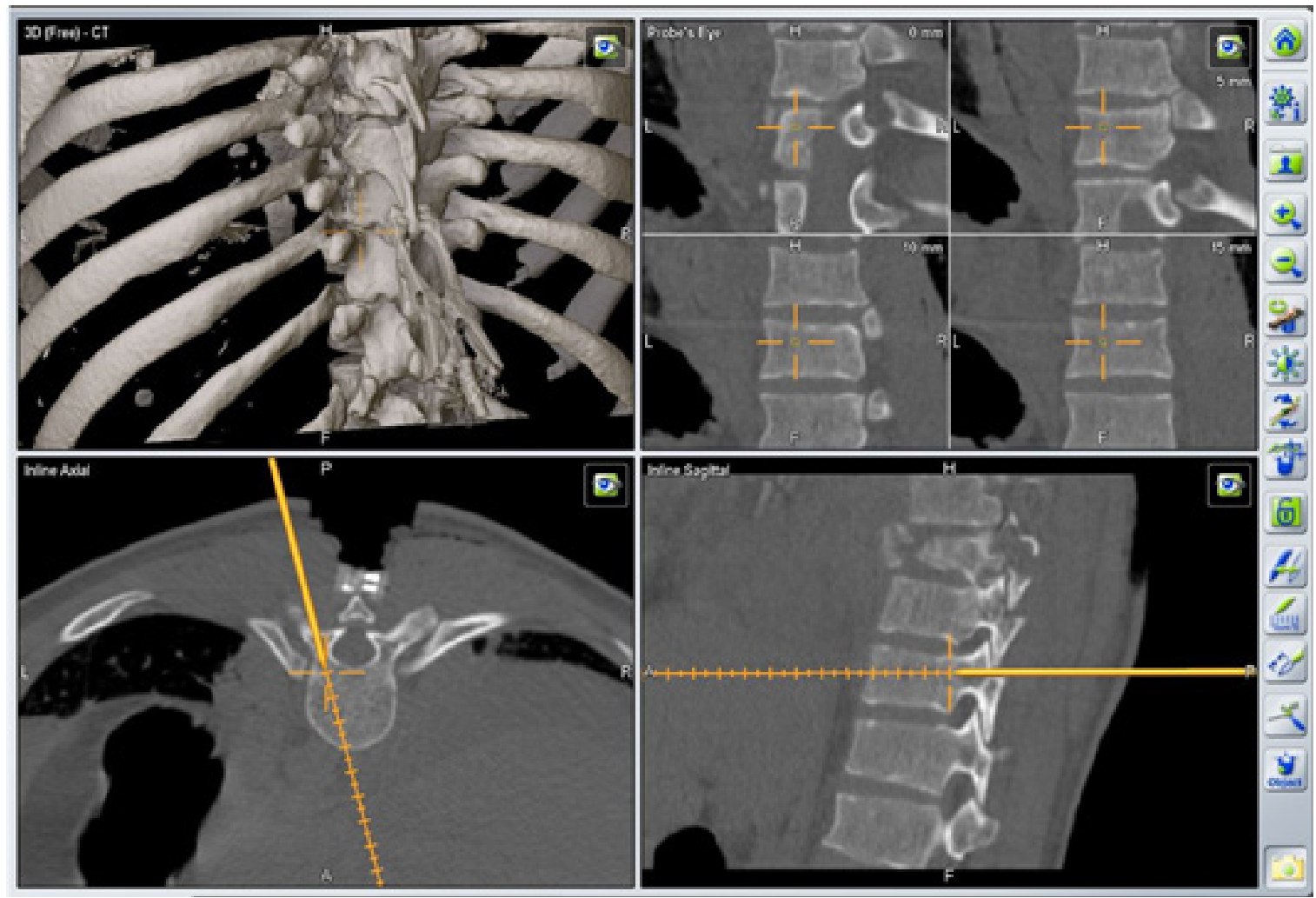

Figure 4: Intraoperative 3D-navigated screw placement trajectory.

\section{Radiologic and Clinical Outcomes}

The postoperative CT-scan demonstrated correction of regional kyphosis with an angle of $32^{\circ}$ (Figure 5). Eleven screws were placed completely within the pedicle unit. Two screws (Th4 and Th7 right) were considered as "in-out-in" with a moderate penetration of the external pedicle wall (3-6mm outside the pedicle boundaries)9, hence there was no indication to reposition these. The patient regained his mobility and started to walk three days following the surgery. An additional thoraco-lumbar corset was placed for a period of 6 weeks. He was discharged 7 days after surgery without any postoperative complications and resumed his professional activity a month later. 


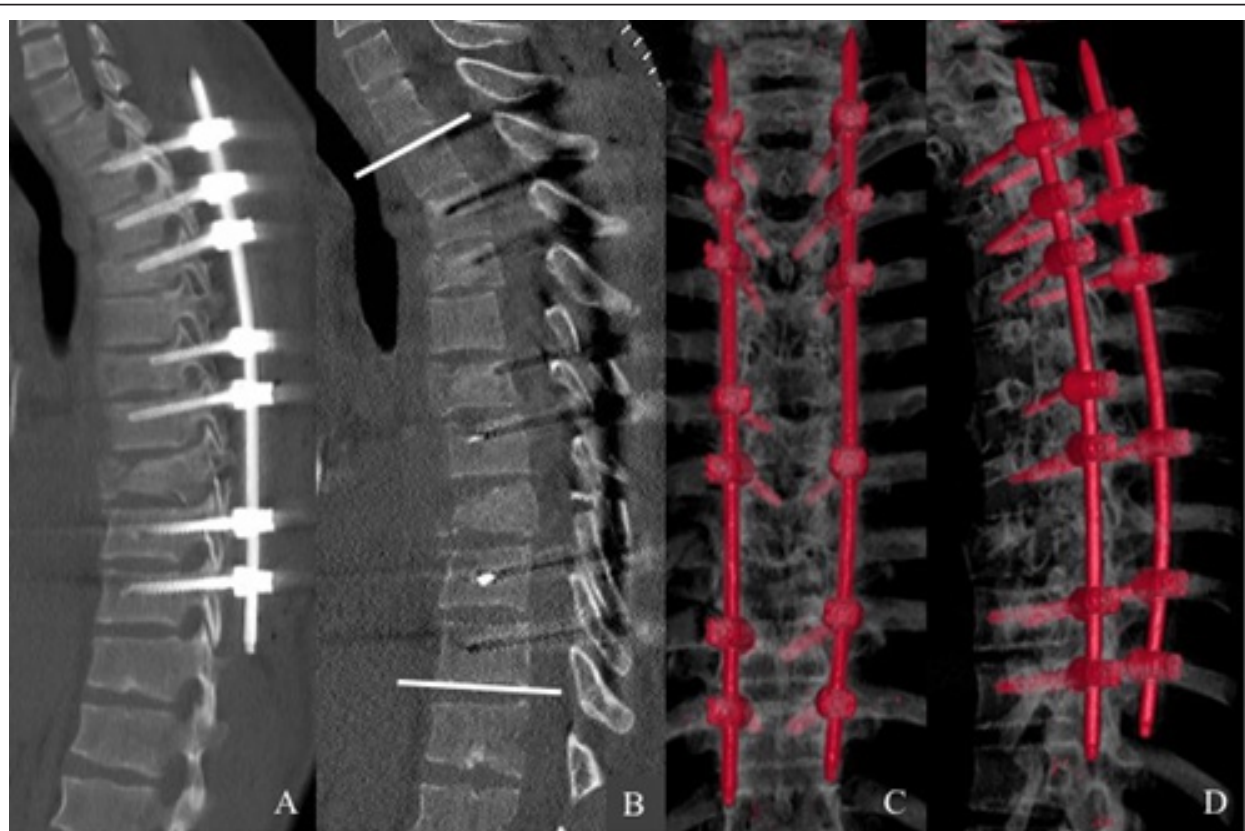

Figure 5: Postoperative sagittal CT-scan reconstruction of the instrumentation from Th2 to Th10. (A) The regional kyphosis was measured with an angle of $32^{\circ}$ (white lines) after the surgery. (B) Posterior (C) and left posterolateral (D) 3D images.

\section{Discussion}

The case reported here is a unique presentation of triple unstable noncontiguous thoracic spine burst fractures without any neurologic lesions. Furthermore, the way he was treated by means of intraoperative minimal invasive navigation despite the multitude and the non-contiguous of these fractures has never been reported in the literature. In case of non-neurologic thoracolumbar fractures, it is more and more commonly admitted that a rapid surgical fixation allows a better management compared to conservative treatment. Recent developments of percutaneous techniques have also optimized the treatment of other comorbidities. It allows early mobilization, decrease the complication rate and reduce the duration of hospitalization, as well as it could be appliable to treat various types of fractures [1,2,4,5,7-9]. Although there are still debates, our experience shows that this is also the case for noncontiguous fractures [1-5]. Some factors such as the important length of fixation, anchoring difficulties on a multi-fractured spine and repercussion on spine mobility should be considered. It is not always possible to restore a perfect spine alignment. Reduction as much as possible of the number of fixed levels is a major concern in order to optimize the clinical outcome and spine range of motion. Yet, when the number of intact segments in between two fractured segments that need surgery is lower than 4 , it is recommended to connect both fixations in one single instrumentation.1 From the very beginning of the medical care, the objective was to multiply anchor points, especially in between fractured level and even in fractured vertebrae in order to reduce the fixation length and maintain spine mobility. This could also increase the rigidity of the instrumentation and reduce the use of transverse connector. Percutaneous pedicular thoracic spine screwing is not an easy procedure for many reasons: the narrowness of the pedicles, radiological opacification generated by the ribs, anatomical modifications due to the trauma and instability of fractured fragments. The use of intraoperative navigation like the Airo system has recently proved his superiority in terms of precision with a correct placement rate of $95-99 \%$ compared to $89-95 \%$ with conventional free hand percutaneous technique $[8,9,11-13]$. Regarding these data, there is no doubt that the 3D-intraoperative navigation technique has the best accuracy. In this specific case, intraoperative navigation has been helpful to securely realize pedicular screwing at many segments and in particular in two fractured vertebrae (Th4 and Th6) 8,9,13. It also allowed the intraoperative control of screws placement and kyphosis correction [8,10-13]. When needed it gives you the possibility of directly replacing misplaced screws and avoid reoperation. However, intraoperative spine navigation remains a difficult and time-consuming procedure that requires a learning curve to be comfortable with $[8,9,11-13]$. There have been concerns about extended surgical time. Recent series show that the instrumentation setup for iCT navigation requires more time, but each screw placement is faster than with free-hand technique and takes about 2 to 3 minutes. One in the other, the total time for the whole procedure seems comparable $[8,9,11,12]$.

Another main hazard during minimal invasive surgery is the radiation exposure that is greater to the surgeon compared with open spine procedures $[9,11]$. The use of intraoperative navigation can decrease the global radiation exposure $[9,11]$. In case of such a long fixation, the medical staff irradiation was significantly reduced. However, patient irradiation is higher with intraoperative CT-scan acquisition than in usual procedure. On the other hand, regarding radiation hazard, each intraoperative acquisition remains lower than a standard diagnostic CT $[9,11,13]$. Two screws were considered as "in-out-in" (Th4 and Th7 right) without 
any consequences for spine stability or neurological damage. This inaccuracy could be explained by biomechanical instability, movements of reference marker during trajectory navigation, instrumentation at a large distance from the tracking device or inaccuracy of Jamshidi needle setting $[8,9,12,13]$. From a technical point of view, some recommendations can be given for the use of intraoperative navigation in long fixation: The use of an additional lateral C-arm fluoroscopy can be easily implemented, even at a later time during surgery, and provide direct radiological information about screws placement or bone fragment displacement $[11,12]$. This information will be correlated by the surgical team with the image-guided navigation and gives a double check when in doubt or in critical regions. In order to optimize the precision, a new CT-scan acquisition should be performed every 4 to 5 segments allowing the repositioning of the reference marker $[10,13]$. The setting of the navigated Jamshidi needle must also be checked frequently, ideally at the start of each pedicular sight [11]. Due to the number of percutaneous tubes needed in very long fixation, it is strongly recommended to start the navigation with the level farthest from the camera in order not to be disturbed by the interposition of tubes between Jamshidi needle and the camera.

\section{Conclusion}

Noncontiguous triple fractures of the thoracic spine are an unusual presentation of polytrauma injury. Long and complex thoracic posterior spine fixation is feasible in a safe way using an intraoperative CT-navigated minimal invasive technique in support $[8-10,12,13]$. The use of intraoperative imaging provides critical information about spine alignment and pedicular trajectories which facilitate screw placement in fractured or misplaced vertebrae [13]. It requires optimal surgical preparation and intervention steps must be followed scrupulously in order to avoid classic traps. Finally, this technique can be labelled as "hybrid" as it combines the use of a midline incision with minimal invasive fixation, and also the use of fluoroscopy in addition to intraoperative navigation.

\section{Acknowledgement}

Patrick Misson provided the conception and design of the article, drafting the article, revised it critically for important intellectual content, and final approval of the version to be submitted. Didier Martin and Abdelhammid Mendili were responsible for the article critically for important intellectual content. Corentin Delhaye provided radiological interpretation and image management. Jean Pirson provided the anesthesia. Cristo Chaskis provided the revised the article critically for important intellectual content and gave final approval of the version to be submitted. No funding to declare.

\section{Conflict of Interest}

The authors declare that there is no conflict of interest.

\section{References}

1. Cho Y \& Kim YG (2019) Clinical features and treatment outcomes of acute multiple thoracic and lumbar spinal fractures: a comparison of continuous and noncontinuous fractures. J Korean Neurosurg Soc 62(6): 700-711.

2. Wittenberg RH, Hargus S, Steffen R, Muhr G, Bötel U (2015) Noncontiguous unstable spine fractures. Spine 27(3): 254-257.

3. Takami M, Okada M, Enyo Y, Iwasaki H, Yamada H, et al. (2017) Noncontiguous double-level unstable spinal injuries. Eur J Orthop Surg Traumatol 27(1): 79-86.

4. Seçer M, Alagöz F, Uçkun O (2015) Multilevel non-contiguous spinal fractures: Surgical approach towards clinical characteristics. Asian Spine J 9(6): 889-894.

5. Kim JK, Moon BJ, Kim SD, Lee JK (2018) Minimal invasive non-fusion technique for the treatment of non-contiguous lumbar burst fractures in young age patient: A case report. Med 97(10): 10-13.

6. Nelson DW, Martin MJ, Martin ND, Beekley A (2013) Evaluation of the risk of non-contiguous fractures of the spine in blunt trauma. J Trauma Acute Care Surg 75(1): 135-139.

7. Giorgi H, Blondel B, Adetchessi T, Dufour H, Tropiano P, et al. (2014) Early percutaneous fixation of spinal thoracolumbar fractures in polytrauma patients. Orthop Traumatol Surg Res 100(5): 449-454.

8. Hubbe U, Kogias E, Vougioukas (2009) Image guided percutaneous trans-pedicular screw fixation of the thoracic spine. A clinical evaluation. Acta Neurochir 151(5): 545-549.

9. Allam Y, Silbermann J, Riese F, Greiner PR (2015) Computer tomography assessment of pedicle screw placement in thoracic spine: Comparison between free hand and a generic 3D-based navigation techniques. Eur Spine J 22(3): 648-653.

10. Citak M, Stubig T, Kendoff D (2010) Navigated minimally invasive thoracolumbar pedicle screw placement with flat panel 3-d imaging a feasibility study. Technol Heal Care 18(2): 101-110.

11. Lian X, Navarro RR, Berlin C (2016) Total 3D Airo® navigation for minimally invasive transforaminal lumbar interbody fusion. Biomed Res Int 5027340.

12. Hecht N, Kamphuis M, Czabanka M (2016) Accuracy and workflow of navigated spinal instrumentation with the mobile AIRO ${ }^{\circ}$ CT scanner. Eur Spine J 25(3): 716-723.

13. Scarone P, Vincenzo G, Distefano D (2018) Use of the AIRO mobile intraoperative CT system versus the 0 -arm for transpedicular screw fixation in the thoracic and lumbar spine: a retrospective cohort study of 263 patients. J Neurosurg Spine 29(4): 397-406.

14. Ushijima T, Kawaguchi K, Matsumoto T (2018) Double non-contiguous fractures in a patient with spondylo-epiphyseal dysplasia with spinal ankylosis treated with open and percutaneous spinal fixation technique: A case report. BMC Res Notes 11(1): 1-5. 